\title{
The gamma-ray sky as seen with HAWC
}

\author{
Petra Hüntemeyer ${ }^{\mathrm{a}}$ \\ Department of Physics, Michigan Technological University, Houghton, MI 49931, USA
}

\begin{abstract}
The High-Altitude Water Cherenkov (HAWC) TeV Gamma-Ray Observatory located at a site about two hours drive east of Puebla, Mexico on the Sierra Negra plateau (4100 m a.s.l.) was inaugurated in March 2015. The array of 300 water Cherenkov detectors can observe large portions of the sky simultaneously and, with an energy range of $100 \mathrm{GeV}$ to $100 \mathrm{TeV}$, is currently one of the most sensitive instruments capable of probing particle acceleration near PeV energies. HAWC has already started science operation in the Summer of 2013 and preliminary sky maps have been produced from 260 days of data taken with a partial array. Multiple $>5 \sigma$ (pretrials) hotspots are visible along the galactic plane and some appear to coincide with known $\mathrm{TeV}$ sources from the H.E.S.S. catalog, SNRs and molecular cloud associations, and pulsars wind nebulae (PWNe). The sky maps based on partial HAWC array data are discussed as well as the scientific potential of the completed instrument especially in the context of multi-wavelengths studies.
\end{abstract}

\section{The HAWC TeV gamma-ray observatory}

The High-Altitude Water Cherenkov (HAWC) telescope is a continuously operating, wide field of view observatory sensitive to gamma rays and cosmic rays in the energy range of $50 \mathrm{GeV}$ to $100 \mathrm{TeV}$. The telescope is capable of performing unbiased searches for transients to initiate multi-messenger observations. After five years of operation it will have accumulated an exposure of about $100 \mathrm{~km}^{2} \mathrm{hr}$ on more than half of the sky. As a wide-fieldof-view instrument the HAWC observatory is particularly well suited to measure large scale and extended structures such as the Fermi bubble structures or diffuse emission from the Galactic plane. HAWC combines the advantage of possessing the large duty cycle and field of view of space telescopes with the sensitivity to $\mathrm{TeV}$ energies. Thereby it provides a complementarity to imaging air Cherenkov telescope like VERITAS, MAGIC, and H.E.S.S., which are characterized by a smaller field of view but better angular resolution.

Construction of the HAWC observatory began in 2012 and was completed in 2015. The observatory is located at $4100 \mathrm{~m}$ a.s.l. and $18^{\circ} 59^{\prime} 41^{\prime \prime} \mathrm{N} 97^{\circ} 18^{\prime} 30.6^{\prime \prime} \mathrm{W}$ at Sierra Negra near Pico de Orizaba in Mexico. It consists of 300 water Cherenkov detector (WCD) stations covering an area of $22,000 \mathrm{~m}^{2}$. Each WCD is $7.3 \mathrm{~m}$ wide and $4.5 \mathrm{~m}$ high and contains $200,000 \mathrm{~L}$ of purified water and four upward-facing photomultiplier tubes (PMT) attached to the bottom of each WCD: one high-quantum efficiency 10-inch Hamamatsu R7081-MOD PMT at the center and three 8-inch Hamamatsu R5912 PMTs at $1.8 \mathrm{~m}$ from the center [1]. Over one year of exposure, the completed HAWC array is expected to be sensitive to pure power law spectra at a level of $5 \times 10^{-13} \mathrm{~cm}^{-2} \mathrm{sec}^{-1}(\sim 50 \mathrm{mCrab})$

\footnotetext{
${ }^{a}$ e-mail: petra@mtu.edu
}

above $2 \mathrm{TeV}$ over $5 \mathrm{sr}(40 \%)$ of the sky [2]. Simulation studies predict an energy dependent angular resolution of between $0.1^{\circ}$ (at highest energies) and $0.8^{\circ}$ (at lowest energies).

The reconstruction of the precise direction of a primary gamma ray relies on properly calibrated relative times of PMT signals in an extensive air shower event originating from the primary particle. The PMT signals are digitized by CAEN V1190 TDCs and the time over threshold relates to the pulse height. The time resolution of the TDCs is $100 \mathrm{ps}$, and the leading and the trailing edges of a voltage pulse are recorded. This allows to compute the timeover-threshold (ToT) of the pulse. The HAWC electronics includes a high and a low voltage threshold to sample pulses. A laser calibration system with controllable light output distributed from the counting house to each WCD via a fiber distribution network is used to measure the leading edges of a pulse signal, i.e. the electronic response time, as a function of light intensity [1]. This system provides a timing calibration that is accurate to better than $1 \mathrm{~ns}$.

\section{Galactic science with HAWC}

HAWC simultaneously views large portions of the gammaray sky and has a duty-cycle near 100\% making it the perfect instrument for measurements of extended and diffuse sources of $\mathrm{TeV}$ gamma-ray emission. In the following, several scientific studies that will benefit from this fact are discussed.

\subsection{Galactic diffuse emission}

The measurement of diffuse $\mathrm{TeV}$ gamma-ray emission probes the density and spectra of cosmic rays (CRs) throughout the Galaxy and therefore allows in principle to distinguish the "sea" of cosmic rays permeating the

This is an Open Access article distributed under the terms of the Creative Commons Attribution License 4.0, which permits unrestricted use, distribution, and reproduction in any medium, provided the original work is properly cited. 


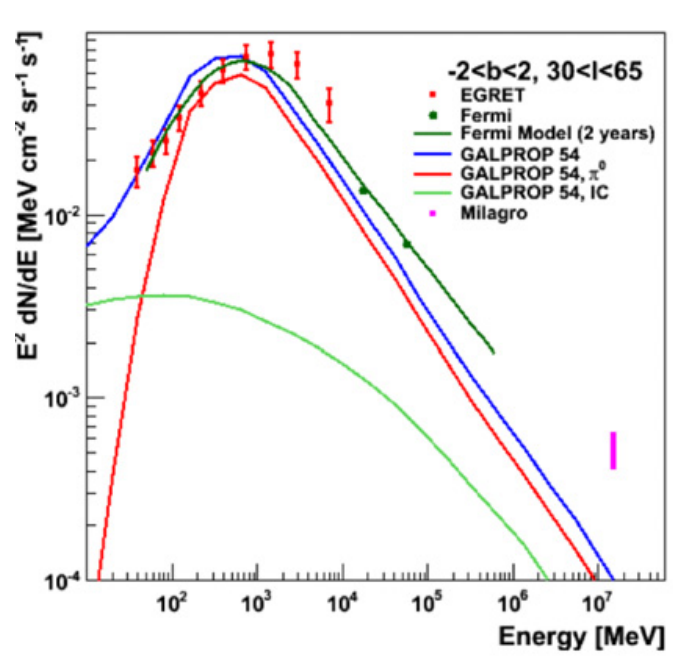

Figure 1. Measured differential fluxes of diffuse gamma-ray emission compared with GALPROP and Fermi model predictions for the inner Galactic plane [20].

whole Galaxy from sites of young CR accelerators. The latter may produce diffuse $\mathrm{TeV}$ gamma-ray emission through the interaction of their freshly realeased CRs with ambient target material, so that diffuse $\mathrm{TeV}$ gamma rays are unique tracers of the transitional energy regime between the Galactic CR background and "young" cosmic rays. Extended or diffuse gamma-ray emission at close to $100 \mathrm{TeV}$ near cosmic-ray acceleration sites would be a sign of CRs that have been accelerated to $10^{15} \mathrm{eV} \mathrm{[3-6].}$

Previous measurements from Milagro and H.E.S.S. support the hypothesis that the cosmic-ray flux is likely to vary throughout the Galaxy [7-11]. Both experiments report diffuse gamma-ray flux excesses with respect to model predictions in the $\mathrm{TeV}$ range in several regions along the Galactic plane. Attempts have been made to explain such excesses by an association with dark matter particles [12-15] but the explanation that the excesses are due to unresolved sources appears more likely $[11,16]$. In contrast, recent results from the Fermi Space Telescope show a good agreement between the conventional assumption of the locally measured CR spectrum and measurements of propagated high energy gamma rays $[17,18]$. Figure 1 shows the result of an analysis of the diffuse emission for an inner Galaxy region of $l \in\left[+30^{\circ},+65^{\circ}\right]$ and $b \in\left[-2^{\circ},+4^{\circ}\right]$ and gammaray energies below $100 \mathrm{GeV}$ using the Fermi Science Tools and the first two years of publicly available data $[19,20]$. For comparison the Milagro measurement and GALPROP prediction in this region is also shown [9,21-23]. The emission Milagro measures from this inner Galaxy region is $4.7 \times$ larger than the GALPROP prediction.

Data from the HAWC telescope will also be used to study potential $\mathrm{TeV}$ gamma-ray emission from the Fermi bubbles. Figure 2 shows the simulated significance that is expected from the north Fermi bubble after three years of data taking with the completed HAWC array assuming a spectral index of $\alpha=1.99$ without a spectral cut-off and using templates acquired from the Fermi Science tools $[19,20]$. Even if the emission is strongly suppressed for energies above about $100 \mathrm{GeV}$ as recent analyses by the

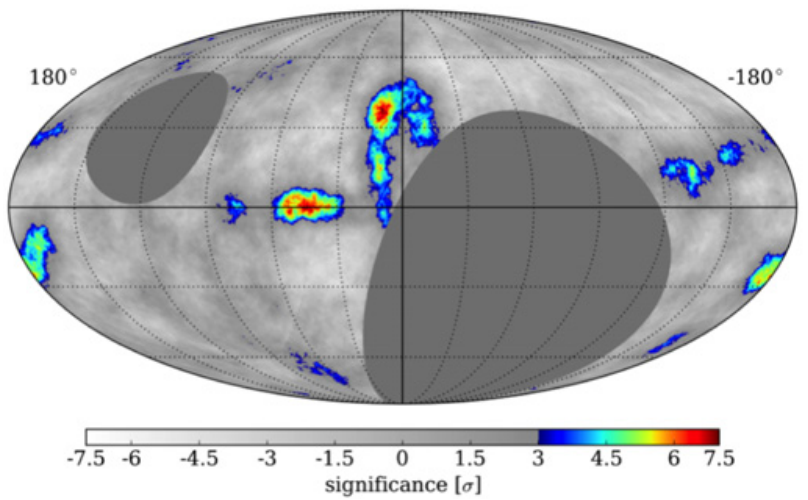

Figure 2. Simulated Fermi bubble significance after three years of data taking and an eight degree smoothing of the sky map [20].

Fermi Collaboration suggest [24], HAWC is currently the only $\mathrm{TeV}$ gamma-ray instrument that will be able to place an upper limit on the emission at very high energies from this very large structure.

\subsection{The Cygnus region}

The Cygnus region is a region of active massive star formation and continues to be studied at different wavelengths, including radio, $\mathrm{X}$-ray, $\mathrm{GeV}$ and $\mathrm{TeV}$ gamma-ray, and in cosmic rays. Between $\mathrm{GeV}$ and $\mathrm{TeV}$ energies, the Cygnus region is the brightest source of diffuse gamma rays in the northern hemisphere. A diverse selection of emission structures have been observed in different wavebands. In addition to diffuse emission at $\mathrm{TeV}$ energies, radiation from molecular clouds, star clusters, pulsars, and a number of gamma-ray sources have been measured $[1,25]$. One of the most exciting recent discoveries is reported by the Fermi Large Area Telescope (LAT): a cavity of enhanced gamma-ray emission with a hard spectrum called the "cocoon" [26]. The interpretation of this object is that particles are accelerated and confined within the cocoon. At $\mathrm{TeV}$ energies the extended Milagro source MGRO J2031+41 appears to be aligned with the cocoon [27]. It also includes the Fermi pulsar 2FGL J2032.2+4126 [1]. The imaging atmospheric Cherenkov telescope (IACT) Whipple reported a point-like emission at the position of the pulsar while IACTs, HEGRA, and MAGIC, reported an extended emission with a radius of about $0.1^{\circ}$.

Figure 3 compares the spectra measured by the various experiments. The emission measured by the IACTs is an order of magnitude weaker than the Milagro flux. The spectra also have different shapes. The spectrum and flux measured by Milagro seems to be compatible with a spectrum of the Fermi cocoon extrapolated to higher energies. Since the angular resolution of the Milagro observatory is significantly larger than that of the IACTs a likely explanation for the difference between Milagro and the IACTs is that the Milagro flux includes both the pulsar wind nebula emission and the diffuse photons emitted by the cocoon region. IACTs on the other hand with their smaller field of view may measure the emission of the smaller pulsar region on top of a diffuse emission background from the cocoon region. 


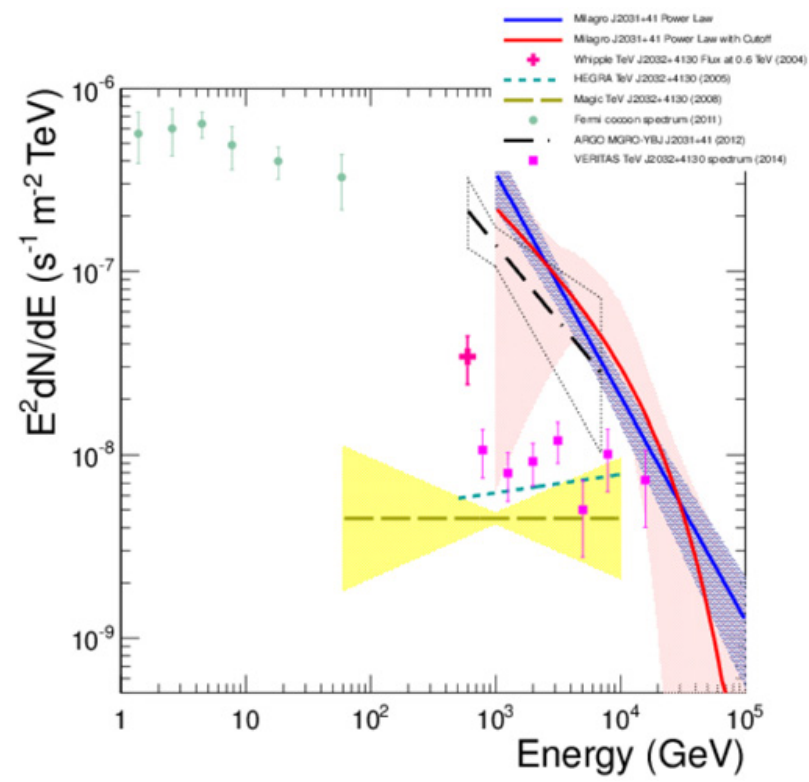

Figure 3. Spectral measurements of the region around MGRO $\mathrm{J} 2031+41$ from various instruments as summarized in [1].

A cosmic-ray acceleration model is favored for the diffuse gamma-ray emission from the Fermi cocoon region. The hardness of the spectrum suggests particles are freshly accelerated and efficiently confined. The Fermi LAT collaboration explained the cocoon emission with protons of a maximum energy from 80 to $300 \mathrm{TeV}$ and electrons from 6 to $50 \mathrm{TeV}$. In addition, it was noted that the Milagro measurement cannot be explained by pure inverse Compton emission alone $[1,26]$. HAWC will be able to reproduce the Milagro map of the Cygnus region from eight years of data taking in a time period that is of the order of weeks, and thus improve spectral and morphological measurements of that region at $\mathrm{TeV}$ energies. This will help with the investigation of the origin and nature of the emission.

Milagro discovered a second source in the Cygnus region, MGRO J2019+37 that was confirmed by VERITAS in a data set based on 75 hours of observation [28]. However VERITAS data indicate that the region may be a complex of several sources. Like the Milagro observatory, which finds that a spectrum with an index of $\alpha=2.0_{-1.0}^{+0.5}$ and an exponential cutoff energy of $29_{-16}^{+50}$ best describes the source, the VERITAS telescope measured a similarly hard spectrum. In the range 1-30 TeV VER J2019+368 is well fit with a power-law model of index $\alpha=1.75 \pm 0.3$. Follow-up multi-wavelength observations of the region, including observations by the HAWC observatory at very high energies should help to clarify the nature of the source.

\subsection{Pulsars and Pulsar Wind Nebulae (PWNe)}

Milagro is the only $\mathrm{TeV}$ instrument that has measured emission from the Geminga region. Though the significance was too low to be classified as source, the Milagro collaboration reported it as a candidate [29]. The horseshoe shaped emission shown in Fig. 4 extends over

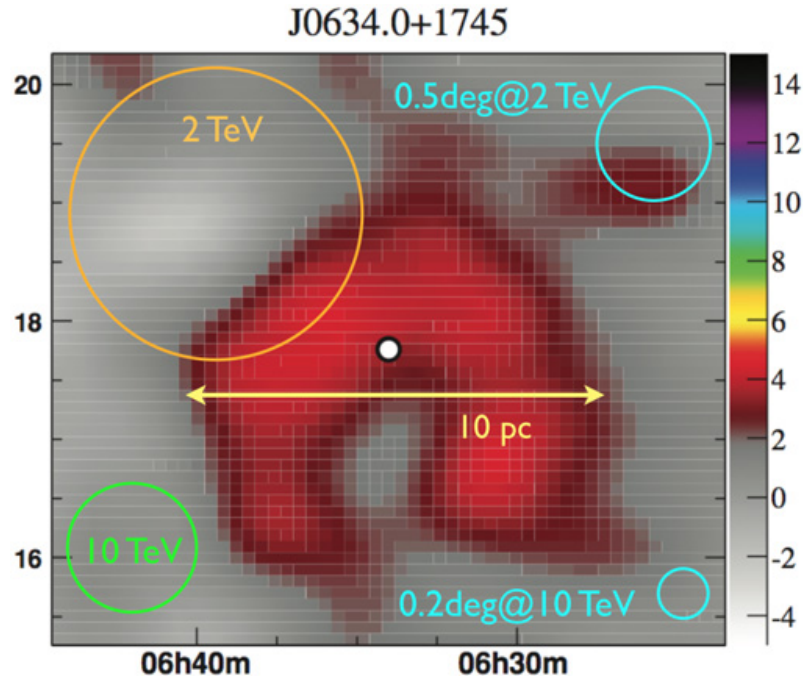

Figure 4. The Geminga pulsar as seen in Milagro data. Overlayed are the source extent in parsec and the energy dependent angular resolution circles of the Milagro (orange and green) and complete HAWC (turquois) observatory [31].

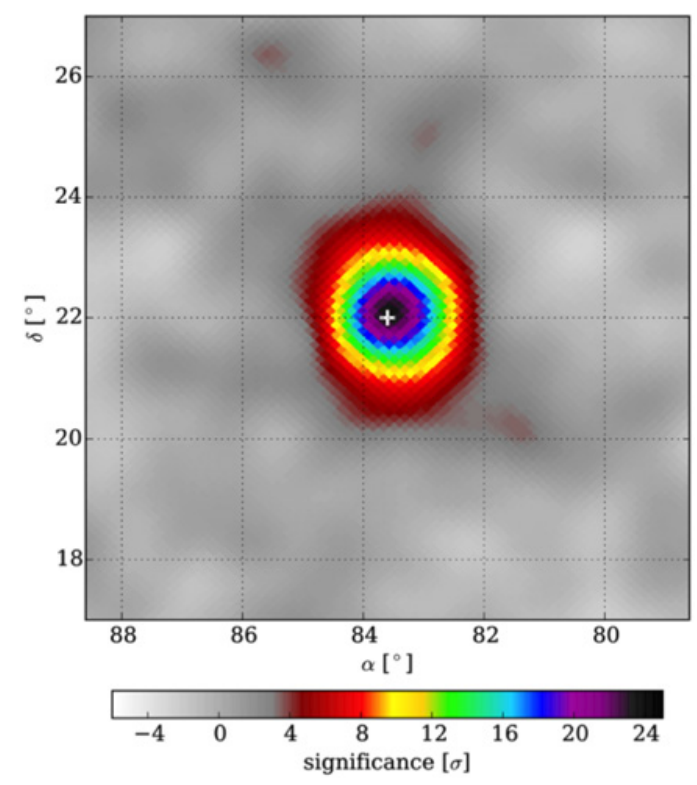

Figure 5. The Crab Nebula in partial HAWC array data (PRELIMINARY).

several degrees, $2.6_{-0.9}^{+0.7}$ o full width at half maximum, corresponding to a distance of $10 \mathrm{pc}$ [30]. This is consistent with observations of more distant pulsar wind nebulae by the H.E.S.S. telescope. The large extent in degrees is due to the relative closeness (169 pc) of Geminga and may explain why Geminga has not been detected by IACTs yet. HAWC data is expected to better constrain the spectrum and flux of this source, which is important given that Geminga and other nearby pulsars are candidates for the explanation of the positron excess measured by Pamela [32]. 

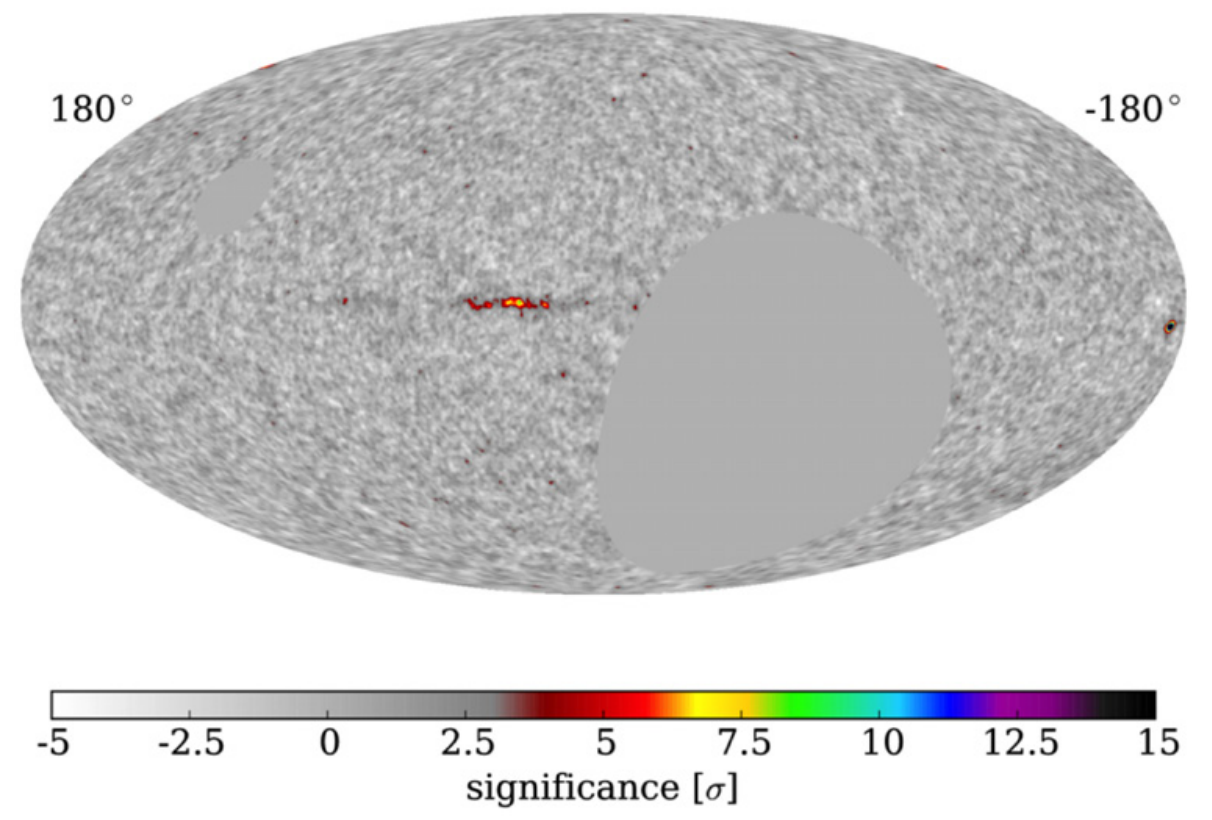

Figure 6. An allsky map based on partial HAWC array data (PRELIMINARY).

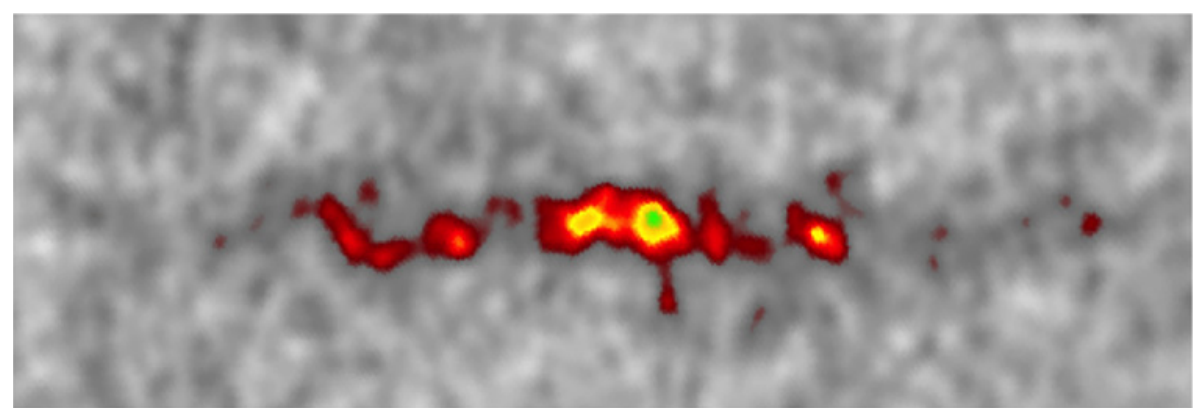

Figure 7. A zoomed view of the inner Galaxy in partial HAWC array data (PRELIMINARY).

\section{Preliminary results from an analysis of partial HAWC array data}

The HAWC observatory began science operation in August 2013 and collected data with a partial array. The data presented here extends to June 2014. During this period data taking was occasionally interrupted resulting in a data set length corresponding to 260 live days. The detector configuration changed from 106 to 133 performanceverified WCDs. The median energy of the analysis is about $2 \mathrm{TeV}$ and varies with declination. Cuts are applied to the data ensuring reconstruction quality and enhancing the signal due to gamma rays. The angular resolution achieved in this data set depends on the size of the detected events and varies between about $0.2^{\circ}$ (for large events) and about $1.4^{\circ}$ (for smaller events). Before the discussion of the presented results it should be duly noted that this is a preliminary analysis, and that systematics affecting the detector pointing and other other effects impacting the data set were still being investigated at the time the following skymaps were produced.

Figure 5 shows a significance map of the Crab Nebula region in the data set described in this paper. The Crab
PWN is the brightest source in the TeV sky, a "standard candle", and often used to compare measurements between different $\mathrm{TeV}$ gamma-ray observatories. In 260 live days of data the Crab PWNe is detected with a pre-trials significance of more than $20 \sigma$ above the isotropic background. This is consistent with the sensitivity anticipated from simulations, after correcting for the observed angular resolutions. The data are divided into ten bins according to the event size (number of PMTs contributing to the reconstructed event), and the Crab Nebula is detected significantly in all the bins.

Figure 6 shows the allsky map based on the data set. In addition to the Crab Nebula, Markarian 421 (barely visible at the north western edge of the map) is detected at $6.5 \sigma$ above the isotropic background. More clearly visible are multiple $>5 \sigma$ detections along the Galactic plane, and some coincide with known $\mathrm{TeV}$ sources from the H.E.S.S. catalog, SNR and molecular cloud associations, and pulsar wind nebulae.

Figure 7 shows a zoomed view of the brightest region along the Galactic plane. The deconvolution and identification of sources in this region is the subject of a forthcoming publication. 


\section{Conclusion}

The HAWC observatory, consisting of 300 WCD stations, is a large-field-of-view, high duty-cycle multi-purpose instrument with high discovery potential as it surveys the sky with $\sim 50$ mCrab sensitivity over 8 sr of the sky at $\mathrm{TeV}$ energies. Data collection with the full detector array that was completed this year is currently on-going. Using data from the array will allow to study the origin, acceleration, and propagation of Galactic cosmic rays, as well as mechanisms present in supernova remnants, pulsar wind nebulae, and binary systems. Already multiple $>5 \sigma$ detections are visible and some coincide with known $\mathrm{TeV}$ sources from the H.E.S.S. catalog, SNR and molecular cloud associations, and pulsars wind nebulae (PWNe). There are a number of other phenomena, such as large and small scale structures in arrival directions of $\mathrm{TeV}$ cosmic rays, dark matter signatures, or extra Galactic sources, such as AGN and GRBs, which can be investigated with HAWC data as well. These are not covered in this paper and are discussed elsewhere.

We acknowledge the support from: the US National Science Foundation (NSF); the US Department of Energy Office of High-Energy Physics; the Laboratory Directed Research and Development (LDRD) program of Los Alamos National Laboratory; Consejo Nacional de Ciencia y Tecnología (CONACyT), Mexico (grants 55155, 105666, 122331, 132197); Red de Física de Altas Energías, Mexico; DGAPA-UNAM (grants IG1004143, IN108713, IN121309, IN115409, IN113612); VIEP-BUAP (grant 161-EXC-2011); the University of Wisconsin Alumni Research Foundation; the Institute of Geophysics, Planetary Physics, and Signatures at Los Alamos National Laboratory; the Luc Binette Foundation UNAM Postdoctoral Fellowship program.

\section{References}

[1] Abeysekara, A., et al., in Proc. 33rd ICRC, Rio de Janeiro, Brazil, arXiv:1310.0074 [astro-ph. IM] (2013)

[2] Abeysekara, A., et al., Astropart. Phys. 50-52, 26 (2013)
[3] Aharonian, and Atoyan, A\&A 309, 917 (1996)

[4] Aharonian, and Atoyan, A\&A 362, 937 (2000)

[5] Casanova et al., PASJ 62, No.5, 1127 (2010)

[6] Gabici, Aharonian, and Casanova, MNRAS 396, 3, 1629 (2009)

[7] Aharonian, et al., Nature 439, 695 (2006)

[8] Atkins, et al., Phys. Rev. Let. 95, 251103 (2005)

[9] Abdo, et al., ApJ 688, 1078 (2008)

[10] Abdo et al., ApJ 658, L33 (2007)

[11] A. Abramowski, et al., Phys. Rev. D 90, 122007 (2014)

[12] Bi, Chen, Wang, and Yuan, ApJ 695, 883 (2009)

[13] Crocker, Bell, Balazs, and Jones, Phys. Rev. D 81, 063516 (2010)

[14] Bertone, Cirelli, Strumia, and Taoso, JCAP 3, 9 (2009)

[15] Meade, Papucci, Strumia, and Volansky, Nuclear Physics B 831, 178 (2010)

[16] Casanova, and Dingus, Astropart. Phys. 29, 63 (2008)

[17] Ackermann, et al., A\&A 538, A71 (2012)

[18] Abdo, et al., ApJ 703, 1249 (2009)

[19] http://fermi.gsfc.nasa.gov/ssc/data/ analysis/, Version v9r32p5 (2013)

[20] H.A. Ayala Solares, Private Communication (2013)

[21] Strong, Moskalenko, and Reimer, ApJ 537, 763 (2000)

[22] Strong, Moskalenko, and Reimer, ApJ 613, 956 (2004)

[23] Strong, Moskalenko, Reimer, Digel, and Diehl, A\&A 422, L47 (2004)

[24] Ackermann, et al., ApJ 793, 64 (2014)

[25] Nolan, P. L., et al., ApJS 199, 3 (2012)

[26] Ackermann, M., et al., Science 334, 1103 (2011)

[27] Abdo, et al., ApJ 753, 159 (2012)

[28] Aliu, et al., ApJ 788, 78 (2014)

[29] Abdo, et al., ApJ 664, L91 (2007)

[30] Abdo, et al., ApJ 700, L127 (2009)

[31] H. Zhou, Private Communication (2014)

[32] Hasan Yüksel, Matthew D. Kistler, and Todor Stanev, Phys. Rev. Lett. 103, 051101 (2009) 